ARTICULO DE INVESTIGACION

\title{
Modelación espacial de área basal y volumen de madera en bosques manejados de Pinus patula y $P$. teocote en el ejido Atopixco, Hidalgo
}

\section{Spatial modeling of basal area and tree volume in managed Pinus patula and $P$. teocote forests in the ejido Atopixco, Hidalgo}

\author{
Isidro Ahmed Cruz-Leyva1, José René Valdez-Lazalde², \\ Gregorio Ángeles-Pérez ${ }^{2}$ \\ y Héctor Manuel de los Santos-Posadas ${ }^{2}$
}

\begin{abstract}
RESUMEN
Se modeló el área basal $\left(A B \mathrm{~m}^{2} / \mathrm{ha}\right)$ y volumen de madera $\left(V O L \mathrm{~m}^{3} / \mathrm{ha}\right)$ de rodales coetáneos de Pinus patula y $P$. teocote localizados en Hidalgo, México, utilizando variables de porcentaje de cobertura arbórea $(C O B)$, índice de área foliar en sitio $\left(L A / 5 \mathrm{~m}^{2} / \mathrm{m}^{2}\right)$ y alométrico $\left(L A I A L \mathrm{~m}^{2} / \mathrm{m}^{2}\right)$, variables cartográficas y de reflectancia obtenidas de una imagen multiespectral del satélite SPOT 5. Para la caracterización de las condiciones ambientales se digitalizó la carta de Efectos Climáticos (escala 1: 250 000) de INEGI y el Modelo Digital de Elevación (MDE) correspondiente a la zona. Se integró un Sistema de Información Geográfica (SIG) constituido por las siguientes capas de información: altitud, pendiente, exposición, temperaturas, precipitaciones, bandas espectrales, y dos grupos de índices de vegetación basados en a) la relación espectral de bandas (NDVI - CTVI) y b) la distancia espectral entre vegetación y suelo desnudo $(P V I-S A V I)$. Con datos de campo y del SIG se ajustaron modelos de regresión lineal múltiple. Para el $A B$ la relación más significativa fue con $L A I A L$, Temperatura Media Anual (TMA), ALTITUD y la Banda 4 de SPOT $\left(P<0,001, \mathrm{R}^{2}=0,9662\right)$. Para el $V O L$ la relación más significativa fue con LAIAL, Temperatura Media Anual $(T M A)$ y $A L T I T U D\left(P<0,001, \mathrm{R}^{2}\right.$ $=0,8856)$.
\end{abstract}

PALABRAS CLAVE:

Inventario forestal, SIG, variables cartográficas, variables espectrales.

\begin{abstract}
Basal area $\left(\mathrm{AB} \mathrm{m}^{2} / \mathrm{ha}\right)$ and tree volume (VOL $\left.\mathrm{m}^{3} / \mathrm{ha}\right)$ were modeled for even-aged Pinus patula and $P$. teocote forest stands located in Hidalgo, Mexico. Variables such as tree crown cover (COB), field recorded Leaf Area Index (LAI5 m²/m²), allometric Leaf Area Index (LAIAL m²/m²); GIS variables and reflectance data derived from a multispectral SPOT 5 image were used as independent variables. Environmental conditions were characterized by digitizing the INEGl's chart of climatic effects (scale 1: 250 000) and processing the Digital Elevation Model (MDE) of the study area. A geographic information system (GIS) was set up with variables such as Elevation, Slope's angle and aspect, Tempe-
\end{abstract}

samed009@gmail.com.

Posgrado Forestal del Colegio de Postgraduados. 56230, Montecillo, Estado de México. valdez@colpos.mx, gangeles@colpos.mx, hmsantos@colpos.mx. 
rature, Precipitation, SPOT derived spectral bands, and two sets of vegetation indices based either on a) the ratio of spectral bands (NDVI and CTVI) or on b) the spectral distance between vegetation and bare soil ( $P V I$ and SAVI). Multiple linear regression models were fitted based on field and GIS data. $A B$ was significantly related to $L A I A L$, Mean Annual Temperature (TMA), ALTITUD and SPOT Band 4 reflectance $(P<$ $\left.0,001, R^{2}=0,9662\right)$. VOL was significantly related to $L A I A L$, Mean Annual Temperature (TMA) and ALTITUD $(\mathrm{P}<$ $\left.0,001, \mathrm{R}^{2}=0,8856\right)$.

KEY WORDS:

Forest inventory, GIS, cartographic variables, spectral variables.

\section{INTRODUCCIÓN}

Una de las razones principales que han impedido evaluar de manera confiable los recursos forestales es el alto costo asociado a los métodos tradicionales de mapeo, tanto en términos monetarios como de tiempo. Por ejemplo, el primer Inventario Nacional Forestal de México requirió 24 años para completarse de 1961 a 1985 (Sorani y Álvarez, 1996). Afortunadamente, la evaluación de los recursos naturales ha cambiado radicalmente en la última década, gracias a la tecnología de la geomática, en particular a los satélites de observación terrestre y los Sistemas de Información Geográfica (Camarero y Gutiérrez, 1999; Wallerman, 2003).

A pesar de que la geomática ha mejorado las metodologías de los inventarios forestales, sus resultados son válidos únicamente para escalas pequeñas (<1:250 000) (Velázquez et al., 2002). Para escalas grandes (>1:25 000) a escala de rodal, la precisión alcanzada a la fecha no es suficiente para propósitos de manejo forestal (Mäkelä y Pekkarinen, 2001). De aquí que los esfuerzos por modelar el comportamiento del volumen de madera (Lee et al., 1995), el área basal (González et al., 2004) o la geometría de claros a escalas grandes (Silbernagel y Moeur, 2001), se encaminen a comprender los efectos de la absorción y reflectancia de las estructura arbórea (hojas y ramas) (McRoberts et al., 2002), las limitaciones de la resolución espacial y espectral de los sensores remotos (Valdez et al., 2006) y a estudiar modelos matemáticos que mejor describan el comportamiento ecológico de las masas forestales (Franco-López et al., 2001; García-García et al., 2006). En especial, la importancia del análisis estadístico en el proceso de interpolación de datos espaciales y la dependencia del valor de un atributo en función de su localización geográfica (Miranda-Salas y Condal, 2003). En este sentido, el objetivo de este trabajo es describir dos variables importantes del bosque, el área basal $(A B)$ y el volumen de madera (VOL) del ejido Atopixco, por medio de técnicas de modelado espacial, con el fin de aportar herramientas que faciliten la administración racional de los recursos forestales.

\section{OBJETIVOS}

Modelar la distribución geográfica del área basal y volumen de madera de las especies Pinus patula y $P$. teocote.

Obtener un inventario forestal del área basal y volumen de madera a través de la modelación espacial.

\section{METODOLOGÍA}

\section{Área de estudio}

El ejido Atopixco tiene una superficie de 1170,97 ha. Se ubica dentro del municipio de Zacualtipán, Hidalgo, entre las coordenadas $20^{\circ} 37^{\prime} 26^{\prime \prime} \mathrm{N}$ y $98^{\circ} 37^{\prime} 48^{\prime \prime} \mathrm{W}$, y $20^{\circ}$ $35^{\prime} 20^{\prime \prime} \mathrm{N}$ y $98^{\circ} 35^{\prime} 23^{\prime \prime} \mathrm{W}$. Este municipio colinda al norte con Tianguistengo, al sur con Metzquititlán, al este con Veracruz, y al oeste con Metztitlán y Xochicoatlán (Figura 
1). Está conformado por pendientes, mesetas y cañones; la altitud media es de $2100 \mathrm{~m}$. El municipio cuenta con tres ríos principales: Pánuco, Zoyatla y Miniahuaco, además de la laguna Chapultepec. Su sustrato geológico data del periodo Cenozoico (terciario superior) y está conformado por rocas ígneas extrusivas del tipo toba ácida (Ts - Ta, al norte) y basalto (Ts - B, al sur). El sustrato edáfico está representado por suelos acrisol órtico (Ao), luvisol crómico (Lc) y feozem háplico $(\mathrm{Hh})$. En la parte norte del ejido el suelo es rico en nutrientes; caso contrario es la parte sur donde el suelo es delgado y pedregoso (EMM, 2000).

En el área predomina el clima templado húmedo $(\mathrm{C}(\mathrm{m}))$ y templado subhúmedo $(\mathrm{C}(\mathrm{w} 2))$ con temperatura media anual entre $12{ }^{\circ} \mathrm{C}$ y $18^{\circ} \mathrm{C}$, temperatura del mes más frío entre $-3{ }^{\circ} \mathrm{C}$ y $18^{\circ} \mathrm{C}$ y temperatura del mes más caliente bajo $22{ }^{\circ} \mathrm{C}$. La precipitación media anual oscila entre $700 \mathrm{~mm}$ y $2050 \mathrm{~mm}$, precipitación del mes más seco menor de 40 $\mathrm{mm}$; lluvias en verano; la lluvia invernal varía entre $5 \%$ y $10,2 \%$ del total anual (2050 mm) (EMM, 2000).

En los bosques manejados del ejido Atopixco se encuentran especies como: Pinus patula, $P$. teocote y en menor abundancia $P$. greggii y $P$. montezumae, estas últimas especies son introducidas. En las áreas de vegetación natural existen especies tales como: Quercus crassipes, $Q$. laurina, Q. excelsa, Q. castanea, Q. rugosa, Cornus disciflora, Viburnum sp., Cleyera theoides, Alnus sp., Arbutus xalapensis, Prunus serotina, Symplocos sp., Ternstroemia sp., Vaccinium leucanthum, Fagus grandifolia subsp. mexicana, entre otras (Ángeles-Pérez, 1995).

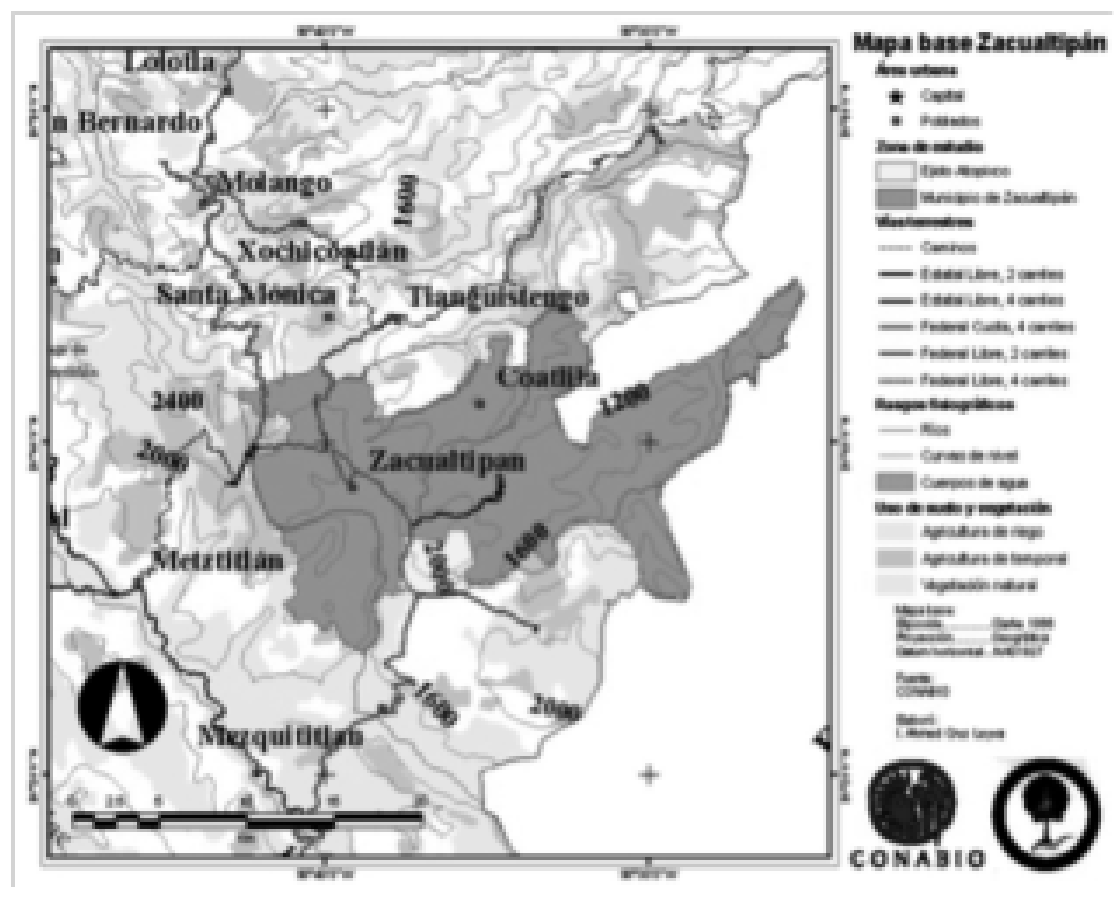

Figura 1. Ubicación de ejido Atopixco, municipio de Zacualtipán de Ángeles, Hidalgo. 


\section{Adquisición y procesamiento en gabi- nete de datos de campo}

Para establecer los límites geográficos del área de estudio y de los diferentes rodales coetáneos que lo integran, se consultó "El plan general de manejo forestal del Ejido Atopixco". Se utilizó además un modelo digital de elevación (resolución 30 m, INEGI).

Se definieron 21 anualidades que corresponden a 38 rodales coetáneos de edades que van de 0 a 26 años. Dentro de cada anualidad se ubicaron al azar 3 sitios circulares de muestreo, cada uno de $400 \mathrm{~m}^{2}$ de superficie. Se marcó el árbol del norte y central, se midió el diámetro a la altura de pecho (DAP) de todos los árboles y la altura total de al menos 5 árboles de DAP variable. Con un GPS Trimble Geoexplorer III se georeferenció cada uno de los 114 sitios muestreados (FAO, 2004).

Para estimar el volumen total de madera de Pinus spp. se empleó la ecuación de Carrillo-Anzurez et al. (2004), misma que fue ajustada para $P$. patula con datos del área de Huayacocotla, Ver., región muy cercana al área de estudio, donde $P$. patula es la especie dominante:

$$
W O L=\exp (-9,7688) \times\left(D A P^{2} \times A L T\right)^{\text {म61 }}
$$

donde: $\mathrm{VOL}=$ Volumen total de madera $\left(\mathrm{m}^{3}\right)$, DAP $=$ Diámetro a la altura del pecho (cm) y ALT = Altura total $(\mathrm{m})$.

El volumen total, en especies hojosas, fue estimado con auxilio de la ecuación ajustada por SAG (1976):

$$
V O L=\exp (-9.80434696) \times D A P^{1.91033696} \times A L T^{1.03262007}
$$

\section{Inventario forestal}

Con la información obtenida en los 114 sitios de muestreo, se estimó el inventario de los recursos maderables en el ejido Atopixco. Para esto se aplicaron tres técnicas de inventariado siguiendo la formulación expuesta por Schreuder et al. (2005): muestreo simple aleatorio (MSA), muestreo estratificado al azar (MEA) y muestreo de regresión (MRR). El propósito fue obtener una estimación base del área basal $(A B)$ y el volumen de madera (VOL) que sirviese de referencia para comparar las estimaciones realizadas con los modelos basados en las variables cartográficas, espectrales y biofísicas.

\section{Procesamiento de la información espectral (SPOT5 HRG)}

Se adquirió una imagen SPOT5 de la Secretaría de Marina a través del Colegio de Postgraduados. La escena fue tomada el 18 de abril de 2006, sus características se reportan en la tabla 1 .

Corregida la geometría y la radiación de la escena (Lillesand y Kiefer, 2001) el siguiente paso fue calcular dos tipos de índices de vegetación (IVs). El primer tipo es conocido como "basados en la pendiente" (Slope-Based VIs), el cual resulta de la combinación de las bandas rojo e infrarrojo cercano. Bajo esta categoría se obtuvieron los siguientes índices: el Índice Vegetación de Diferencia Normalizada (NDVI) y el Índice de Vegetación Transformado (corregido) (CTVI) (Eastman, 2001).

El segundo tipo de IVs utilizados se denomina "basados en la distancia" (Distance-Based VIs). Éstos se utilizan en ambientes donde la vegetación no es densa y es necesario separar la reflectancia del suelo de la reflectancia de la vegetación. 
Tabla 1. Parámetros de la escena SPOT5

\begin{tabular}{|c|c|c|c|c|}
\hline Fecha & \multicolumn{2}{|c|}{$2006-04-18$} & \multicolumn{2}{|c|}{$17: 05: 10.5$} \\
\hline Instrumento & \multicolumn{4}{|c|}{ HRG 2} \\
\hline Número de bandas & \multicolumn{4}{|c|}{4} \\
\hline $\begin{array}{l}\text { Indicador de banda } \\
\text { espectral }\end{array}$ & HI1: Verde & HI2: Rojo & HI3: IR Cercano & HI4: IR Medio \\
\hline Longitud de onda $(\mu \mathrm{m})$ & $0,50-0,59$ & $0,61-0,68$ & $0,78-0,89$ & $1,58-1,75$ \\
\hline Número de ganancia & 7 & 7 & 5 & 4 \\
\hline $\begin{array}{l}\text { Calibración absoluta de } \\
\text { ganancias }\left(\mathrm{W} / \mathrm{m}^{2} / \mathrm{sr} / \mu \mathrm{m}\right)\end{array}$ & 2,139452 & 2,853960 & 1,738550 & 8,225268 \\
\hline Ängulo de orientación & \multicolumn{4}{|c|}{12,264436 grados } \\
\hline Ángulo de incidencia & \multicolumn{4}{|c|}{20,187256 grados } \\
\hline Ángulos del sol & \multicolumn{2}{|c|}{ Azimut: 111,469109 } & \multicolumn{2}{|c|}{ Elevación: 66,197548} \\
\hline Filas & \multicolumn{4}{|c|}{7302} \\
\hline Columnas & \multicolumn{4}{|c|}{7784} \\
\hline
\end{tabular}

Para esto se hace una correlación entre las bandas roja e infrarroja, utilizando como factor de corrección en pendiente $(, 0)$ a las zonas de suelo desnudo $(P V I)$ o, en su caso, transformando las bandas roja e infrarrojo en radianza absoluta (SAVI) (Eastman, 2001). Bajo esta categoría se obtuvieron dos índices: el Índice Perpendicular de Vegetación ( $P V I)$ y el Índice de Vegetación Ajustado a la Pendiente del Suelo (SAVI).

\section{Generación del sistema de información geográfica}

Para caracterizar las condiciones ambientales del área de estudio, se generó (en el paquete IDRISI KILIMANJARO) un Sistema de Información Geográfica (SIG) a partir del modelo digital de elevación (MDE) de resolución espacial de $30 \mathrm{~m}$ y de las cartas Edafológica y Geológica (escala 1:50000) y de Efectos climáticos (escala 1:250000) para los periodos de lluvias (mayooctubre) y secas (noviembre-abril) de INEGI. La información de las cartas se digitalizó por medio de una tableta y del paquete ARCGIS. Las cartas digitales obtenidas fueron transformadas a formato raster con resolución semejante a la del MDE. A partir del MDE se obtuvo la capa de elevación sobre el nivel medio del mar (ALTITUD) y se construyeron las capas de pendiente (PENDIENTE) y exposición (EXPOSICIÓN) del terreno. Las cartas de isolíneas climáticas (isotermas e isoyetas) se interpolaron mediante el módulo INTERCON del paquete IDRISI KILIMANJARO. De esta manera se obtuvieron las cartas de temperatura media anual (TMA), temperatura máxima (TMAX), temperatura mínima (TMIN), precipitación media anual (PMA), precipitación en lluvias (PMAMO) y precipitación en secas (PMANA). Se consideraron además las variables biofísicas índice de área foliar en sitio ( $L A / 5)$, índice de área foliar alométrico (LAIAL) y cobertura arbórea (COB) (Aguirre, 2007).

\section{Predicción de $A B$ y VOL mediante regresión lineal múltiple (Stepwise)}

Los datos de campo (inventario) correspondientes a área basal $(A B)$ y volumen de madera ( $V O L)$ se empataron con las nueve variables cartográficas del medio físico (información ambiental), con los datos espectrales obtenidos de la escena SPOT5 (Bandas 1-4), con los datos espectrales transformados en índices de vegetación -IV'S (NDVI, CTVI, PVI y SAVI) y con los índices de área foliar. Esta base de datos permitió ajustar, por separado, para las variables $A B$ y $V O L$, modelos de regresión lineal múltiple $(R L M)$ de la forma general: 


$$
\begin{aligned}
\mathrm{AB}, \mathrm{VOL} & =\beta_{0}+\beta_{1} \mathrm{X}_{1}+\beta_{2} \mathrm{X}_{2}+\ldots+\beta_{9} \mathrm{X}_{9}+\beta_{10} \mathrm{~B}_{1}+\ldots+\beta_{13} \mathrm{~B}_{4}+\beta_{\mathrm{i}} \mathrm{IV}+\varepsilon_{i} \\
\mathrm{AB}, \mathrm{VOL} & =\beta_{0}+\beta_{1} C O B+\beta_{2} \mathrm{X}_{1}+\beta_{3} \mathrm{X}_{2}+\ldots+\beta_{10} \mathrm{X}_{9}+\beta_{11} \mathrm{~B}_{1}+\ldots+\beta_{14} \mathrm{~B}_{4}+\beta_{\mathrm{i}} \mathrm{IV}+\varepsilon_{i} \\
\mathrm{AB}, \mathrm{VOL} & =\beta_{0}+\beta_{1} L A I 5+\beta_{2} \mathrm{X}_{1}+\beta_{3} \mathrm{X}_{2}+\ldots+\beta_{10} \mathrm{X}_{9}+\beta_{11} \mathrm{~B}_{1}+\ldots+\beta_{14} \mathrm{~B}_{4}+\beta_{\mathrm{i}} \mathrm{IV}+\varepsilon_{i} \\
\mathrm{AB}, \mathrm{VOL} & =\beta_{0}+\beta_{1} L A I A L+\beta_{2} \mathrm{X}_{1}+\beta_{3} \mathrm{X}_{2}+\ldots+\beta_{10} \mathrm{X}_{9}+\beta_{11} \mathrm{~B}_{1}+\ldots+\beta_{14} \mathrm{~B}_{4}+\beta_{\mathrm{i}} \mathrm{IV}+\varepsilon_{i}
\end{aligned}
$$

Los modelos genéricos arriba descritos se ajustaron mediante el procedimiento STEPWISE (Pindyck y Rubinfeld, 1981) para predecir los valores promedio de $A B$ $y$ VOL que se esperaría alcance un sitio (píxel) donde las condiciones ambientales sean $\mathrm{X}_{1}, \mathrm{X}_{2}, \ldots, \mathrm{X}_{9}$; las propiedades espectrales sean $\mathrm{B}_{1}, \mathrm{~B}_{2}, \ldots, \mathrm{B}_{4}$; y los IV's sean los indicados, al igual que las variables biofísicas consideradas. Con el fin de identificar y corregir inconsistencias estadísticas en los modelos, además de las pruebas ANOVA $(P<0,05)$ y del factor de inflación de varianza (VIF < 1 o 5), se buscaron observaciones aberrantes (OA) con relación al conjunto de datos a predecir $\left(Y_{i}\right)$ y del conjunto de variables predictoras $\left(X_{i}\right)$ (Belsley et al., 2005).

Finalmente, los cuatro modelos que presentaron mejor ajuste, tanto para $A B$ como para $V O L$, se usaron para predecir la distribución espacial de tales variables en la totalidad de la superficie del ejido Atopixco. Para ello se resolvieron los modelos en cada uno de los pixeles que conforman el ejido.

\section{RESULTADOS Y DISCUSIÓN}

A través del inventario se obtuvo un valor promedio de área basal $(A B)$ de 19,96 $\mathrm{m}^{2} /$ ha bajo muestreo simple aleatorio (MSA) y de 22,54 $\mathrm{m}^{2} /$ ha bajo muestreo aleatorio estratificado (MEA). El volumen de madera (VOL) promedio obtenido fue de $118,89 \mathrm{~m}^{3} / \mathrm{ha}$ (MSA) y de 140,69 m³/ha para las 21 anualidades o estratos (MEA). Se estimó un inventario final de $42019,11 \mathrm{~m}^{3}$, para el Ejido Atopixco, con una variación estimada en $\mathrm{VOL}$ de \pm 2 218,59 $\mathrm{m}^{3}$ (5,28\%) (Tabla 2).

Para mejorar la precisión porcentual (B/y) del inventario en $\mathrm{VO}$, se procedió a aplicar los estimadores de muestreo de regresión (MRR). Si se utiliza solamente como valor de $\mu_{x}$ al promedio de los datos, el promedio estimado es igual al valor de la media, obtenida bajo MSA $\left(118,89 \mathrm{~m}^{3} / \mathrm{ha}\right)$. Sin embargo, se tiene un estimador alterno de $\mu_{\mathrm{x}}$ que es el valor de $A B$ promedio obtenido bajo MEA y que es mucho más preciso que el obtenido bajo MSA. Usando este valor como $\mu_{\mathrm{x}}$ para el estimador de regresión se tiene que la media bajo MRR es $\mathrm{Y}_{\text {reg }}=5,55\left(138,8 \mathrm{~m}^{3} / \mathrm{ha}\right)$ (Tabla 3).

Tabla 2. Estimadores por sitio de $400 \mathrm{~m}^{2}$ en muestreo simple aleatorio (MSA) y estratificado aleatorio (MEA).

\begin{tabular}{lcccc}
\hline ESTIMADORES & \multicolumn{2}{c}{ ÁREA BASAL $\left(\mathrm{m}^{2}\right)$} & \multicolumn{2}{c}{ VOLUMEN $\left(\mathrm{m}^{3}\right)$} \\
\hline & MSA & MEA & MSA & MEA \\
\hline Media & 0,7985 & 0,90 & 4,76 & 5,63 \\
Varianza media estimada & 0,0021 & 0,00033 & 0,13 & 0,02 \\
Límite superior de IC & 0,89 & 0,94 & 5,47 & 5,92 \\
Límite inferior de IC & 0,71 & 0,86 & 4,04 & 5,33 \\
Error permisible porcentual (B/y) & $\mathbf{1 1 , 5 3 \%}$ & $\mathbf{4 , 0 6 \%}$ & $\mathbf{1 4 , 9 4 \%}$ & $\mathbf{5 , 2 8 \%}$ \\
Amplitud del IC a 95\% $(\mathrm{B})$ & 0,09 & 0,04 & 0,71 & 0,30 \\
Inventario total $(\mathrm{T})$ & 5961,76 & 6730,69 & 35507,37 & 42019,11 \\
Media Alfa & 0,04 & 0,05 & 0,24 & 0,28 \\
Tamaño de muestra, $\cdot(\mathrm{n})$ & 568,27 & 65,07 & 908,02 & 100,78 \\
Alfa $(\alpha)$ & 0,05 & 0,05 & 0,05 & 0,05 \\
\hline
\end{tabular}


Tabla 3. Estimadores de volumen por sitio de $400 \mathrm{~m}^{2}$, en muestreo por regresión (MRR).

\begin{tabular}{lc}
\hline ESTIMADORES DE REGRESIÓN & $\begin{array}{c}\text { VOLUMEN } \\
\left(\mathrm{m}^{3}\right)\end{array}$ \\
\hline b (pendiente) & 7,38 \\
Correlación VOL vs. AB & 0,96 \\
Media & 5,55 \\
Covarianza de XY (Syx) & 1,29 \\
Varianza media regresión (SmedReg) & 0,01 \\
Límite superior de IC & 4,97 \\
Límite inferior de IC & 4,54 \\
Error permisible porcentual (B/y) & $\mathbf{3 , 8 0} \%$ \\
Amplitud del IC a 95\% (B) & 0,21 \\
Inventario total (T) & 41454 \\
\hline
\end{tabular}

Este resultado sugiere que asumir MSA tiende a subestimar considerablemente las existencias totales en volumen de madera y es mucho menos preciso, siendo los estimadores de MEA y MRR más adecuados para estimar el inventario.

\section{Predicción de $A B$ mediante regresión lineal múltiple}

La tabla 4 presenta las variables significativas y los valores de los estimadores que describen los cuatro modelos de regresión lineal múltiple ajustados para predecir el $A B$ mediante el procedimiento Stepwise. El coeficiente de determinación múltiple $\left(R^{2}\right)$ de los modelos propuestos es moderadamente alto, sus valores varían explicando de $73,1 \%$ a $96,62 \%$ de la variabilidad.

Cabe resaltar que el modelo 3 es el que presenta la mayor capacidad predictiva $\left(R^{2}=0,96\right)$ mediante la inclusión de la variable biofísica LAIAL y las variables cartográficas ALTITUD y TMA. Los modelos 1 a 4 (Tabla 4) se usaron para predecir los valores de $A B$ a escala de píxel.

\section{Modelado espacial del área basal $(A B)$}

El modelado espacial se obtuvo resolviendo los modelos para la totalidad de los pixeles que conforman el área de estudio, construyendo así la distribución espacial del $A B$ en el ejido Atopixco. Por ejemplo, la figura 2 muestra tal distribución con base en el modelo etiquetado como Modelo 1, donde la relación entre $A B$ y $C O B$ es positiva y alta (64,9\% correlación semiparcial). La COB es un reflejo de la densidad. Márquez-Linares y Álvarez-Zagoya (1995) muestran que en Pinus cooperi de diferentes clases de copa, hay una relación negativa entre el diámetro y la densidad conforme la competencia aumenta. Es decir, la competencia tiene un efecto importante en la pendiente de la relación tamaño (diámetro)-densidad, a partir del punto en el cual la competencia se hace más intensa (individuos intermedios).

También la alta densidad, en rodales jóvenes, puede producir árboles con bajo porcentaje de ramas vivas a lo largo del tronco, coberturas del rodal muy altas y fustes cónicos (Black y Abrams, 2005). Esto puede ser captado por las fotos hemisféricas (Jonckheere et al., 2004) y explicaría porqué rodales jóvenes presentan altos valores de cobertura arbórea (Figura 2).

En la figura 3 se muestra la distribución espacial del $A B$ pero ahora con base en el modelo 2 (Tabla 4), aquí la relación más alta del $A B$ se da con el índice de área foliar ( $L A / 5$ ), misma que es positiva (68\% correlación semiparcial).

En los cuatro modelos ajustados para predecir $A B$, destaca la variable índice de área foliar alométrico (LAIAL) (Figura 4), es ésta la que más aportó a la $\mathrm{R}^{2}$ ajustada (Tabla 4). A diferencia de $L A 15$, el LAIAL está altamente relacionado con el área basal, pues su estimación depende del diámetro normal (DAP) (Cano-Morales et al., 1996; Figueroa-Navarro, 2010). 
Tabla 4. Significancia $(P<0,001)$ del modelo de regresión lineal múltiple para estimar área basal $(A B)$ y estimadores de los parámetros $\beta$ i.

?

R-cuadrada

R-ajustada

\begin{tabular}{|c|c|c|c|c|}
\hline & $\begin{array}{c}\text { Estimador } \\
\text { parámetro } \\
\text { i }\end{array}$ & $\operatorname{Pr}>|t|$ & $\begin{array}{l}\text { Corr., tipol } \\
\text { semiparcial } \\
\text { cuadrado }\end{array}$ & FIV \\
\hline Intercepto & 8,222 & 0,0001 & & 0 \\
\hline $\mathrm{COB}$ & 0,016 & 0,0001 & 0,64984 & 1,67 \\
\hline LAI 5 & & & & \\
\hline LAIAL & & & & \\
\hline PENDIENTE & $-0,018$ & 0,0029 & 0,02113 & 1,1 \\
\hline ALTITUD & & & & \\
\hline ALTITUD2 & & & & \\
\hline TMA & $-0,565$ & 0,0001 & 0,09731 & 1,68 \\
\hline TMAX & & & & \\
\hline TMAX2 & & & & \\
\hline PMA & & & & \\
\hline PMAMO & & & & \\
\hline PMAMO2 & & & & \\
\hline PVI & $-0,054$ & 0,0468 & 0,00912 & 1,01 \\
\hline B4 & & & & \\
\hline
\end{tabular}

\section{Modelo 3}

R-cuadrada

R-ajustada

\begin{tabular}{|c|c|c|}
\hline \multicolumn{3}{|c|}{0,9662} \\
0,9649
\end{tabular}

Modelo 2

\begin{tabular}{|c|c|c|c|}
\hline & $\begin{array}{l}0,8058 \\
0,7938 \\
\end{array}$ & & \\
\hline $\begin{array}{c}\text { Estimador } \\
\text { parámetro } \\
\text { i }\end{array}$ & $\operatorname{Pr}>|\mathrm{t}|$ & $\begin{array}{c}\text { Corr., tipo I } \\
\text { semiparcial } \\
\text { cuadrado }\end{array}$ & FIV \\
\hline 299,52 & 0,004 & & 0 \\
\hline 0,39 & 0,0001 & 0,68 & 1,82 \\
\hline$-0,01$ & 0,0296 & 0,01 & 1,24 \\
\hline$-0,29$ & 0,0051 & 0,0001 & $>5$ \\
\hline 0,0001 & 0,0052 & 0,03 & $>5$ \\
\hline$-0,49$ & 0,0001 & 0,08 & 3,2 \\
\hline$-0,05$ & 0,0453 & 0,01 & 1,02 \\
\hline
\end{tabular}

Mo delo 4

\begin{tabular}{|c|c|c|c|}
\hline & $\begin{array}{l}0,7313 \\
0,7117\end{array}$ & & \\
\hline $\begin{array}{c}\text { Estimador } \\
\text { parámetro } \\
\text { i }\end{array}$ & $\operatorname{Pr}>|\mathrm{t}|$ & $\begin{array}{l}\text { Corr., tipo I } \\
\text { s emiparcial } \\
\text { cuadrado }\end{array}$ & FIV \\
\hline 1177,91 & 0,0001 & & 0 \\
\hline$-0,576$ & 0,0001 & 0,18 & $>5$ \\
\hline 0,0001 & 0,0001 & 0,07 & $>5$ \\
\hline$-58,827$ & 0,0001 & 0,05 & $>5$ \\
\hline 1,656 & 0,0001 & 0,001 & $>5$ \\
\hline 0,183 & 0,0001 & 0,29 & $>5$ \\
\hline$-0,17$ & 0,0001 & 0,001 & $>5$ \\
\hline 0,00002 & 0,0001 & 0,13 & $>5$ \\
\hline
\end{tabular}

$\mathrm{COB}=$ Cobertura arbórea, LAI5 = Índice de área foliar en sitio, LAIAL = Índice de área foliar alométrico, PENDIENTE = Pendiente del terreno, ALTITUD = Altitud sobre el nivel medio del mar, TMA $=$ Temperatura media anual, $T M A X=$ Temperatura máxima, $P M A=P r e c i-$ pitación media anual, $\mathrm{PMAMO}=$ Precipitación media anual Mayo-Octubre, PVI = Índice perpendicular de vegetación, B4 = Banda 4 . 


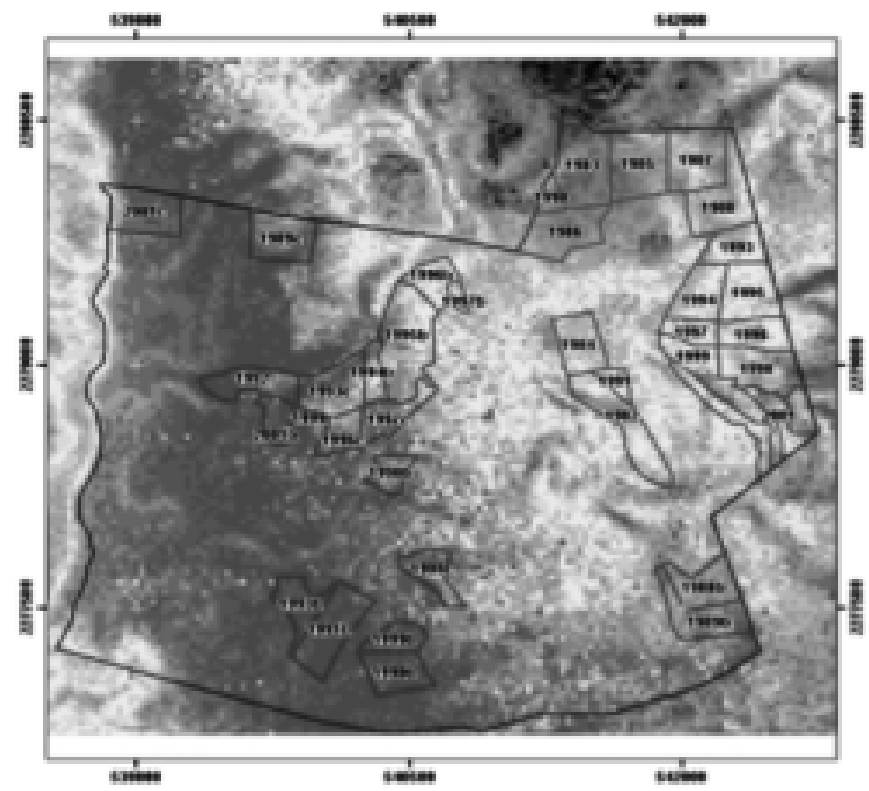

AREA BASAL (m2/ha)

1000-0.57

$0.58-125$

126, 192

193.254 $255 \cdot 3.16$ $13.17-3.74$ 13.75 .4 .36 $4.97 \cdot 5.08$ $504-570$ 1571.6 .32 633.694 695.752 $753-800$ $18.10 \cdot .67$ $1868-9.24$ $925-9.91$ $1992 \cdot 1071$ $1079 \cdot 12.16$

Figura 2. Distribución del área basal ( $\mathrm{m}^{2} / \mathrm{ha}$ ) en el ejido Atopixco, Hidalgo con base en el Modelo 1 (Tabla 4).
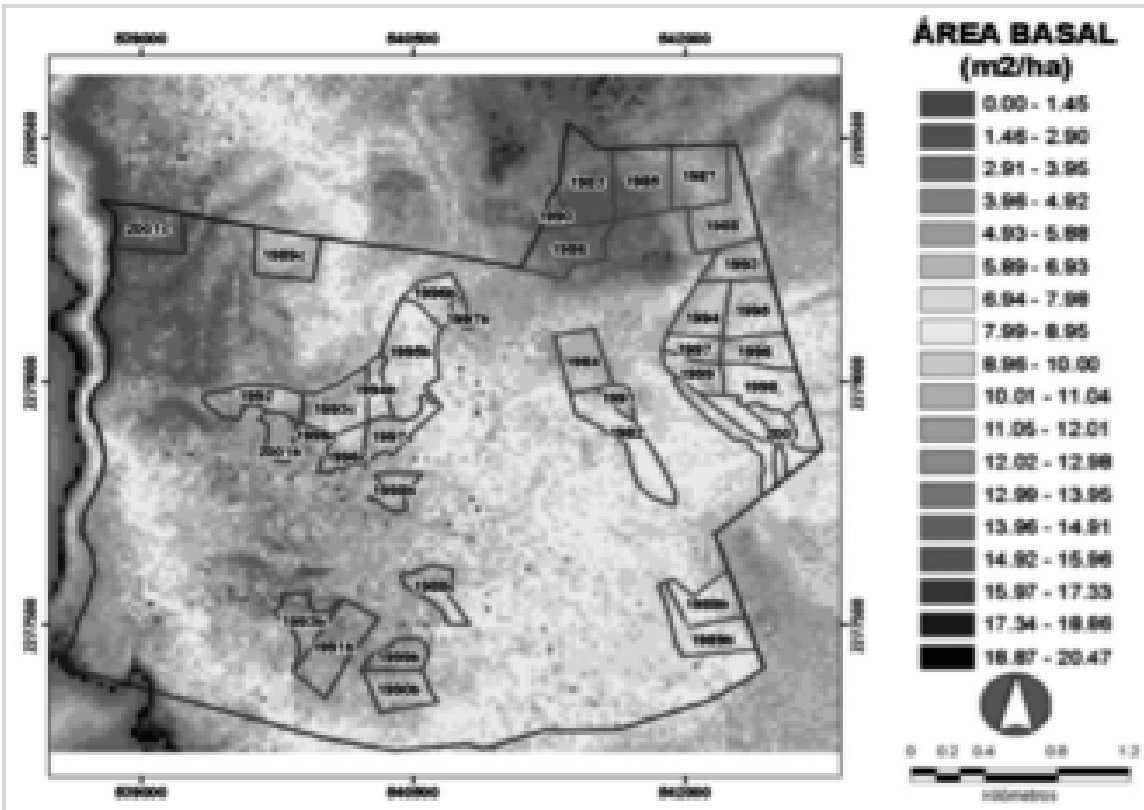

Figura 3. Distribución del área basal ( $\mathrm{m}^{2} / \mathrm{ha}$ ) en el ejido Atopixco, Hidalgo con base en el Modelo 2 (Tabla 4). 
La relación entre el $A B$ y $L A I A L$ puede explicarse como una extensión de la relación que existe entre el tejido vascular (xilema y floema) y la superficie foliar. Según Barrantes y Gracia (1989), el área foliar específica de una hoja (medida en $\mathrm{cm}^{2} / \mathrm{mg}$ ) varía con la altura del árbol. Las hojas situadas a mayor altura (más expuestas a la radiación solar) presentan un área específica menor que las halladas en la parte baja de la copa. En otras palabras, el área foliar es proporcional a la superficie transversal del tronco, por el cual pasa el agua necesaria para abastecerlo y que circula desde las raíces hacia las hojas. Este es un fenómeno muy importante que tiene varias implicaciones; pues no solo relaciona la cantidad de área basal con el índice de área foliar, también muestra que la disponibilidad de agua influye en la estructura arbórea.
En todos los modelos, las variables cartográficas ALTITUD, TMA, PMA y PMAMO siempre se hacen presentes. Mención aparte merecen las variables de reflectancia en la banda B4 y el PVI. En el Modelo 3 (Tabla 4), el coeficiente de la variable $B 4$ es negativo, esto significa que la cantidad de área basal aumenta conforme disminuye la reflectancia en el infrarrojo cercano (B4). En un estudio con plantaciones de Eucalyptus y Pinus, Lencinasa y Mohr-Bell (2007) determinaron una correlación significativa $(-0,58$ y $-0,79)$ entre los valores de la $B 4$ y la edad de dichas plantaciones. Estimaron la edad con un error medio de 2,2 años en eucaliptos y 3,5 años en pinos. Esa relación inversa, entre valores espectrales y la edad para plantaciones de coníferas, también fue reportada por Sivanpillai et al. (2006). Tal fenómeno se explica por el agua de las hojas que absorbe la luz roja,
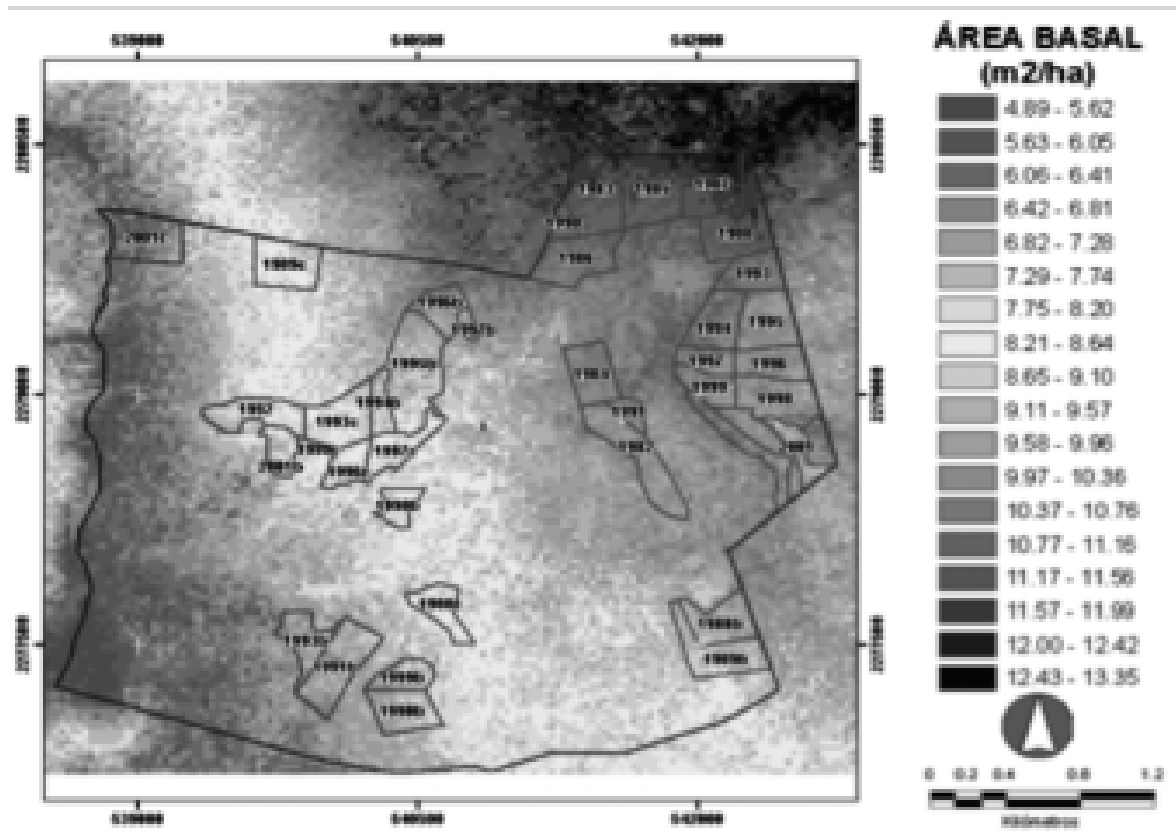

Figura 4. Distribución del área basal $\left(\mathrm{m}^{2} / \mathrm{ha}\right)$ en el ejido Atopixco, Hidalgo con base en el Modelo 3 (Tabla 4). 
mientras que las demás estructuras celulares reflejan la mayor parte de la radiación infrarroja (B4). El incremento en área basal está acompañado por un aumento en el índice de área foliar alométrico (LAIAL) y por tanto una mayor absorción del infrarrojo medio (B4), razón que expone la relación negativa de $A B-B 4$ (Figura 4, Tabla 4).

De acuerdo con Ante et al. (2005), en general los índices de vegetación que toman la razón entre la reflectancia del suelo y la vegetación (por ejemplo SAVI, $P V I$, entre otros) son mejores para discriminar tipos de cobertura vegetal, en zonas con contrastes ambientales, que los índices de vegetación basados en la razón de bandas rojo-infrarrojo (NDVI, CTVI, entre otros). Es de llamar la atención que el coeficiente de PVI (Modelo 2) sea negativo, en tal caso habría que explorar su relación con la variable LAI5 (Tabla 4).

De lo anterior, Gilabert et al. (2002) escriben "el $P V I$ se puede citar como un índice que presenta, dentro del rango de variación, una dependencia lineal con respecto al índice de área foliar ( $L A l)$, y al NDVI, como aquél en el que se observa más claramente la saturación". Para valores intermedios -en densidad de vegetación- la dispersión de la radiación por parte de la masa vegetal puede disminuir la influencia del suelo, en este caso los índices de vegetación tradicionales (como el $N D V I$ ) presentan los valores más altos, indicando una eficacia menor para normalizar la perturbación introducida por la señal del suelo. Se manifiesta la limitación de estos índices en zonas que presentan vegetación dispersa (es el caso del ejido Atopixco). El PVI parece funcionar mejor, probablemente porque define con suficiente precisión la línea del suelo (Figura 3).

Si bien las variables cartográficas ALTITUD, TMA, PMA y PMAMO se hacen presentes en los modelos 1,2 y 3 , es en el Modelo 4 (Figura 5), donde cobran relevancia. Como su nombre lo indica, son variables obtenidas de información cartográfica, lo cual puede ser una virtud o una desventaja (Trotter y Dymond, 1997). En este sentido Miranda-Salas y Condal (2003) afirman que los atributos altitud y área basal $(A B)$ satisfacen la condición de variables interpolables (modelado espacial) y que el proceso de interpolación no puede ser exitoso si primero no se consideran los resultados obtenidos de un análisis estadístico preliminar de los datos a interpolar. Entonces se debe ser cuidadoso al considerar variables cartográficas en la modelación espacial.

Como se aprecia en la figura 5 las variables cartográficas (Tabla 4) describen un rango de valores para $A B$ que van de $12,31 \mathrm{~m}^{2} / \mathrm{ha}$ a $16,09 \mathrm{~m}^{2} / \mathrm{ha}$. Es un rango estrecho en comparación con los modelos que incluyen a las variables biofísicas $C O B$ e índices de área foliar ( $L A I 5$ y $L A I A L)$. Además se hace evidente el peso que tiene la precipitación y la temperatura sobre la modelación espacial del $A B$ (Figura 5). Esto indicaría que los modelos que sólo contemplan variables cartográficas son menos eficaces para la modelación espacial de variables biofísicas ( $C O B$ y $L A I)$ o dasométricas ( $A B$ ) (Zurita-Milla, 2000). Pero no es así, el mayor problema de utilizar tales variables es su construcción. Por ejemplo, las capas raster de temperatura y precipitación fueron obtenidas de cartas escala 1:250 000. Con una escala tan pequeña es complicado realizar operaciones de interpolación, aun cuando se utilice una base raster de $30 \mathrm{~m}$ x $30 \mathrm{~m}$ de resolución, el resultado es poca variabilidad espacial. Aún con ésta y otras desventajas se pudieron obtener coeficientes de correlación moderadamente altos (Tabla 4). De mejorar la calidad de la información contenida en mapas se daría un enorme avance en la modelación de variables dasométricas (Lillesand y Kiefer, 2001; Eastman, 2001). 

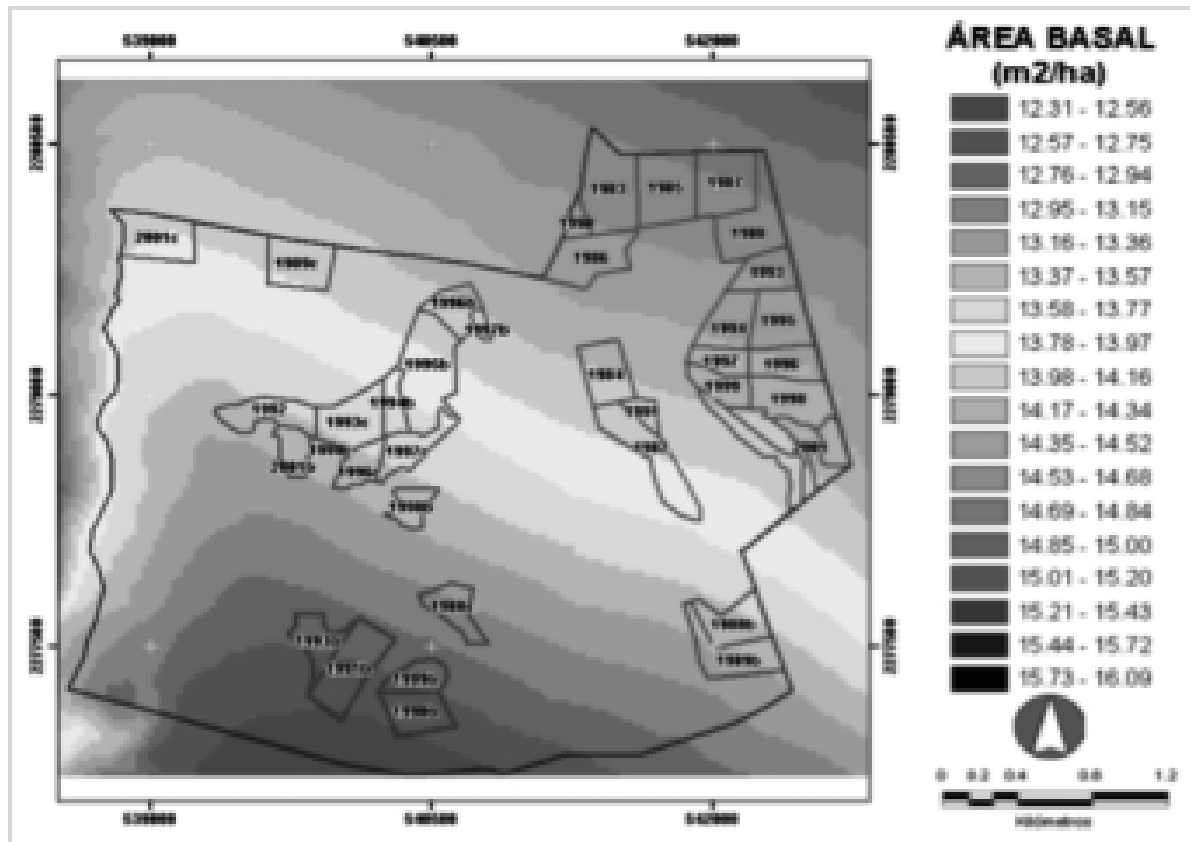

Figura 5. Distribución del área basal ( $\mathrm{m}^{2} / \mathrm{ha}$ ) en el ejido Atopixco, Hidalgo con base en el Modelo 4 (Tabla 4).

Sin olvidar lo mencionado arriba, queda de manifiesto la utilidad de emplear variables cartográficas como altitud, precipitación y temperatura para describir la distribución del $A B$. Los coeficientes de altitud y temperatura guardan una relación positiva con el incremento de $A B$. Esto concuerda con el trabajo de Camarero y Gutiérrez (1999) quienes describieron el patrón espacial de un ecotono de bosque dominado por Pinus uncinata, a través de variaciones en altitud, tipo de sustrato, y cobertura de herbáceas y arbustos. La descripción del ecotono permitió discernir la posible respuesta de las poblaciones de $P$. uncinata ante cambios ambientales. Por su parte, Sánchez y López-Mata (2003) al realizar un análisis sobre la vegetación y los factores que influyen en su estructura y distribución a lo largo de la Sierra Nevada, notaron que la relación (correlación canónica) entre vegetación y altitud-precipitación fue positiva hasta un cierto rango, mientras que la temperatura presenta coeficientes canónicos negativos. La temperatura en esa zona templada se asocia a sitios de cobertura vegetal seca y menor cantidad de precipitación. La misma tendencia fue observada en el ejido Atopixco. Un hecho importante destacado por Sánchez y López-Mata (2003) fue que la distribución de las especies era continua a lo largo del gradiente. En consecuencia el patrón en la estructura y distribución de las comunidades arbóreas está definido por el gradiente altitudinal (temperatura y precipitación) y por las propiedades del suelo (pendiente del terreno, materia orgánica, cationes, nutrimentos y la profundidad del horizonte $\mathrm{A}$ y $\mathrm{O}$ ). Elementos que podrían valorarse en futuras investigaciones para el ejido Atopixco. 


\section{Comparación de las estimaciones de área basal: inventario vs modelación}

De los cuatro modelos propuestos para estimar el área basal $(A B)$, el Modelo 2 (que incluye $L A 15$ ) se aproximó más a la estimación del inventario total. El valor de $A B$ obtenido para el ejido por MEA fue de $22,54 \mathrm{~m}^{2} / \mathrm{ha}$, mientras que la estimación mediante el modelo 2 fue de $20,47 \mathrm{~m}^{2} /$ ha, es decir, con una diferencia en área basal de $2 \mathrm{~m} 2 /$ ha; un margen menor si se compara con la estimación mediante MSA (2,6 $\left.\mathrm{m}^{2} / \mathrm{ha}\right)$. Estos resultados concuerdan con los presentados por Aguirre (2007) quien observó que el $A B$ fue el parámetro forestal que registró el error cuadrático medio (RECM) más pequeño para pinos. Un dato muy importante, pues el área basal siempre fue la variable respuesta que mejor ajuste tuvo en relación con las variables independientes. Por otro lado, destaca el resultado obtenido con el Modelo 4, el cual está integrado únicamente por variables cartográficas. A pesar de tener la menor $R^{2}$ ajustada $(0,7117)$ fue el segundo modelo que arrojó estima- ciones más cercanas al inventario por MSA (tablas 4 y 5 ).

\section{Estimación de VOL mediante regresión lineal múltiple}

La tabla 6 presenta las variables significativas y los valores de los estimadores que describen los modelos de regresión lineal múltiple ajustados para predecir el VOL. Estos modelos fueron la base para predecir la distribución espacial del $\mathrm{VOL}$ en la superficie forestal del ejido Atopixco. Los cuatro modelos obtenidos (modelos 5-8) ponen de manifiesto la importancia de utilizar variables biofísicas LAI5 y LAIAL; y cartográficas como TMA, PENDIENTE, ALTITUD, PMA y PMAMO en la estimación del VOL. Merece la pena destacar también los valores obtenidos de los coeficientes de determinación múltiple $\left(R^{2}\right)$, mismos que oscilan entre 0,72 y 0,88 ; este último se considera alto, representando una capacidad explicativa del $88 \%$.

Tabla 5. Comparación de las estimaciones de área basal $\left(\mathrm{m}^{2}\right)$ con inventario tradicional y con inventario por SIG. Intervalos de confianza (IC) a 95\% de significancia estadística.

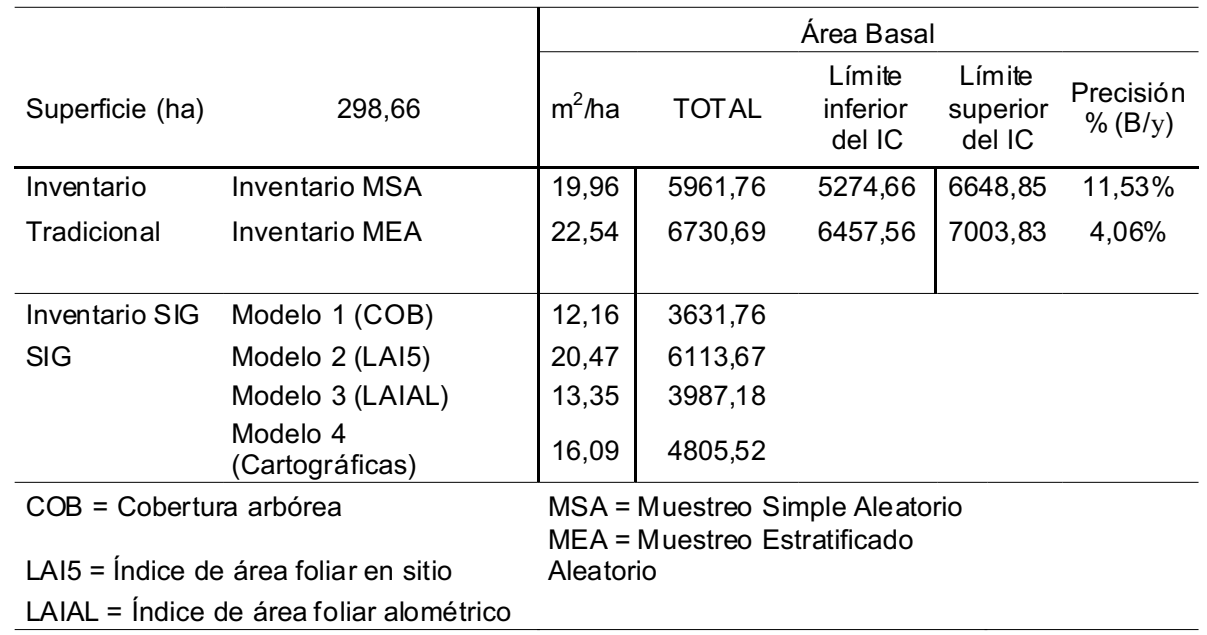


Tabla 6. Significancia $(P<0,001)$ del modelo de regresión lineal múltiple para estimar VOL y estimadores de los parámetros $\beta$ i.

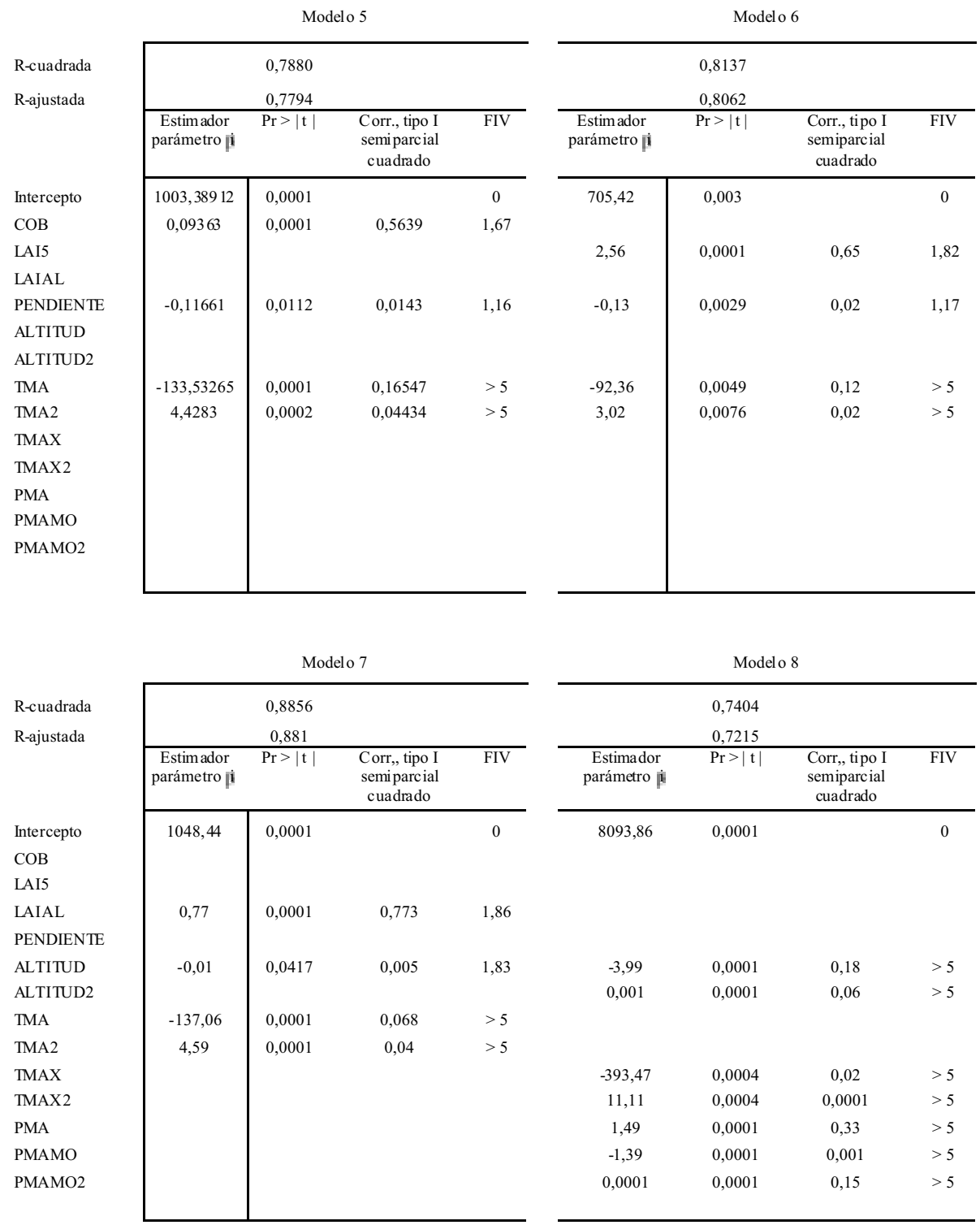

$\mathrm{COB}=$ Cobertura arbórea, LAI5 = Índice de área foliar en sitio, LAIAL = Índice de área foliar alométrico, PENDIENTE = Pendiente del terreno, $A L T I T U D=$ Altitud sobre el nivel medio del mar, TMA = Temperatura media anual, $T M A X=$ Temperatura máxima, $P M A=P r e c i-$ pitación media anual, PMAMO = Precipitación media anual Mayo-Octubre. 


\section{Modelado espacial del volumen de madera ( $V O L)$}

En la figura 6 se muestra la distribución del $V O L$ en el ejido Atopixco con base en el Modelo 5 (Tabla 6). Es de destacar la importancia de las variables $C O B$ y $T M A$ en la distribución.

La temperatura se correlaciona negativamente con el VOL, es decir, a mayor temperatura menor cantidad de madera. Regionalmente esto puede asociarse a la disponibilidad de humedad (precipitación) y cómo ésta influye en la actividad del árbol y de algunos microorganismos. Como menciona LiétorGallego (2002), en bosques de Abies pinsapo, las condiciones extremas de temperatura y humedad del suelo retardan la actividad de los microorganismos relacionados con el ciclado del $\mathrm{N}$ (mineralización y nitrificación). Al final del invierno y principios del otoño, coincidiendo con los máximos de producción de hojarasca y con la recuperación de condiciones de temperatura y humedad favorables, se incrementan los niveles de $\mathrm{N}$ mineral en el suelo y los microorganismos son más efectivos para liberar nutrientes. Un fenómeno que podría explicar en parte la distribución arbórea en el ejido Atopixco.

La $C O B$ es la variable que más aportó a explicar el comportamiento del $\mathrm{VOL}$ (Tabla 6). Esta variable es sencilla de medir en campo, sin embargo, al momento de modelar se observa que la PENDIENTE influye sobre la $C O B$ y por ende sobre el VOL, aún cuando su coeficiente de correlación semiparcial sea bajo. Como explica Valdez-Lazalde et al. (2006) una opción adecuada para estimar

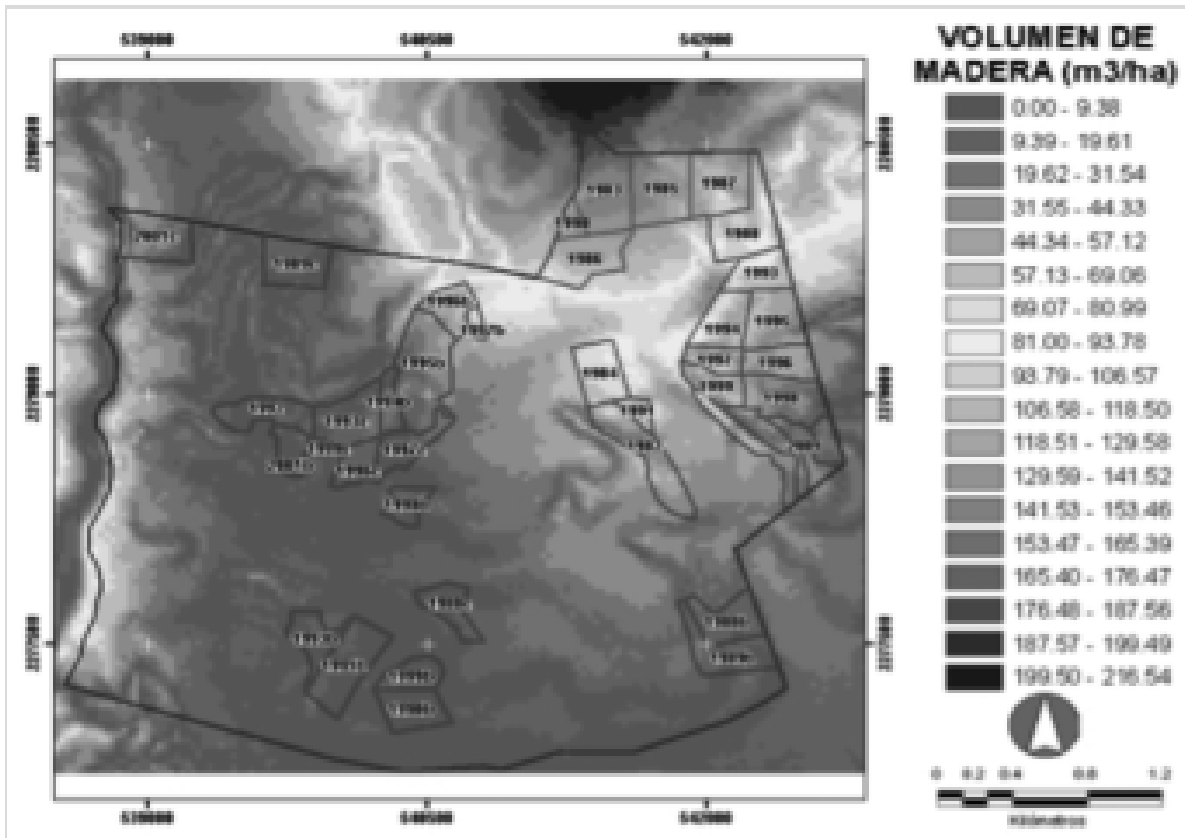

Figura 6. Distribución del volumen de madera $\left(\mathrm{m}^{3} / \mathrm{ha}\right)$ en el ejido Atopixco, Hidalgo con base al Modelo 5 (Tabla 6). 
la cobertura de copas, cuando las áreas tienen condiciones fisiográficas accidentadas, es contar las sombras registradas (en imágenes de satélite) como áreas de vegetación; contrariamente, en sitios de poca inclinación las sombras efectivamente son suelo sin cubierta vegetal. Esto de forma indirecta puede revelar porqué la PENDIENTE influyó sobre el VOL; las sombras formadas por las pendientes debieron contarse como

En el Modelo 6 (Figura 7) la PENDIENTE también produce un efecto positivo sobre la cantidad de VOL. Sin embargo, su contribución al modelo (Tabla 6) no se compara con la aportación del $L A / 5$. Tanto la $C O B$ como el $L A / 5$ dependen de la geometría de copas, de la arquitectura de hojas y ramas, así como de la cantidad de luz que éstas dejan pasar hasta la cámara (Frazer et al.,
1999), no obstante hay una mejor relación lineal del $V O L$ y $L A I 5$ que del $V O L$ y $C O B$, razón por la cual el coeficiente de determinación múltiple del Modelo 6 es mayor que el coeficiente del Modelo 5 (Tabla 6).

Al igual que en el Modelo 5, también en el Modelo 6 (Figura 7) se hace presente la TMA, misma que puede asociarse con la distribución de la humedad. De hecho, todos los modelos muestran una tendencia hacia el aumento creciente del VOL, desde el suroeste hacia el noreste; lo cual coincide con el cambio del régimen climático de una zona árida (Valle de Metztitlán) hacia una zona templada (ejido La Mojonera). Esto viene apoyado por la presencia de la variable ALTITUD que representa el gradiente altitudinal (Figura 7).

De los modelos ajustados para estimar VOL, el Modelo 7 (Figura 8) es el

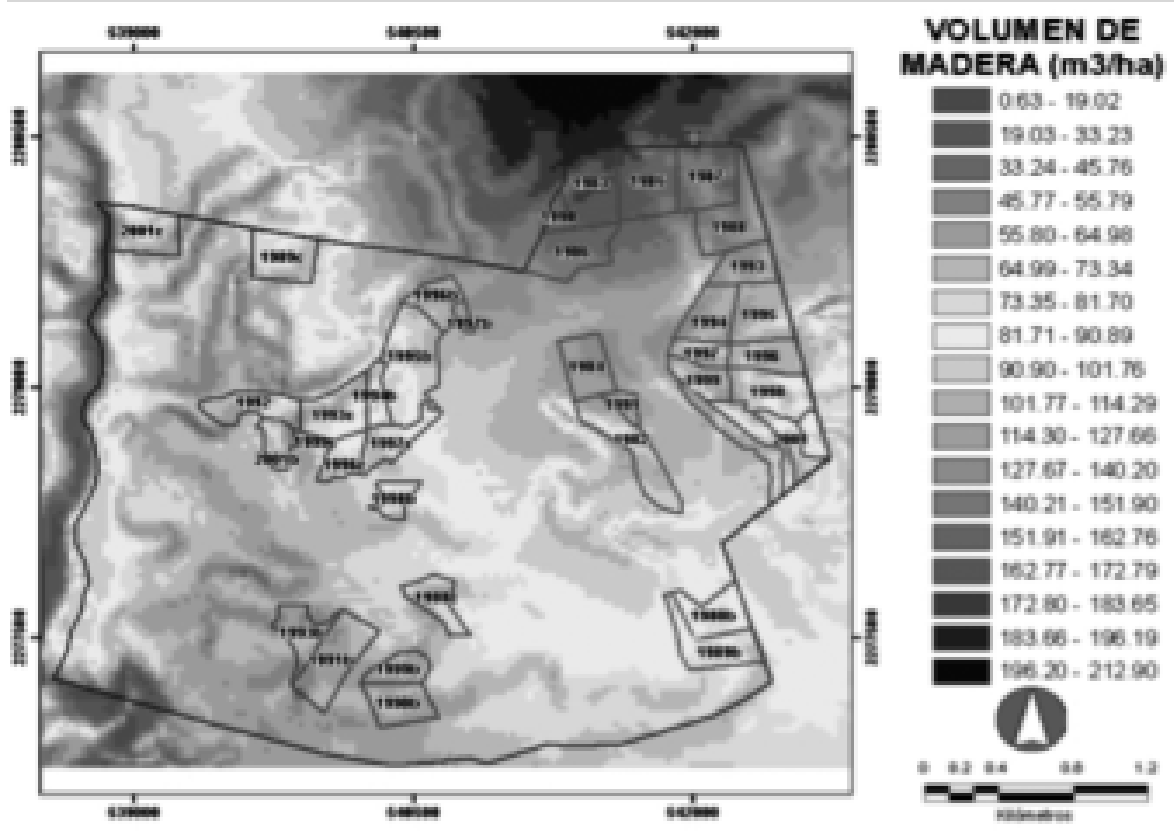

Figura 7. Distribución del volumen de madera $\left(\mathrm{m}^{3} / \mathrm{ha}\right)$ en el ejido Atopixco, Hidalgo con base al Modelo 6 (Tabla 6). 
que presenta el mayor coeficiente de determinación (Tabla 6). En este caso, es evidente la alta relación existente entre la variable $L A I A L$ y el $V O L$ a través del $A B$. En este modelo, el gradiente climático (temperatura y elevación sobre el nivel del mar) ejerce un marcado efecto sobre la distribución del VOL. Se esperaría que el modelo espacial que incluye al LAIAL fuera mejor que los modelos que incluyen como variables dependientes a la $\angle O B$ y al $L A I 5$, dado que su $\mathrm{R}^{2}$ fue la más alta de todos los modelos. No obstante, en términos generales, el Modelo 7 vuelve a mostrar la tendencia de aumento en $\mathrm{VOL}$ desde el suroeste hacia el noreste (ejido La Mojonera). En este sentido, es posible perfeccionar las predicciones en $V O L$ si por un lado se aumenta la precisión de las variables cartográficas y por otro se halla un modelo matemático que emule la complejidad del paisaje. Esto es apoyado por García-García et al. (2006) y McRoberts et al. (2002) quienes comprobaron que los modelos espaciales son susceptibles de mejoras si se reduce la heterogeneidad del bioma, lo cual hace que la relación entre variables forestales y variables radiométricas no sea tan alta como las observadas en otros medios forestales más homogéneos.

De los cuatro modelos ajustados para predecir el VOL, el Modelo 8 (Tabla 6) generado a partir de variables cartográficas presenta el menor coeficiente de correlación $\left(R^{2}=0,74\right)$. Esto se ve reflejado en el intervalo del $V O L$ que va de 99,9 $\mathrm{m}^{3} /$ ha a $126,6 \mathrm{~m}^{3} /$ ha (Figura 9), en comparación al Modelo 6 tiene un intervalo de $0,6 \mathrm{~m}^{3} / \mathrm{ha}$ a $213 \mathrm{~m}^{3} / \mathrm{ha}$. Al igual que en el modelo de área basal (Figura 5), los coeficientes para ALTITUD, TMAX, $P M A$ y $P M A M O$ indican que la cantidad de

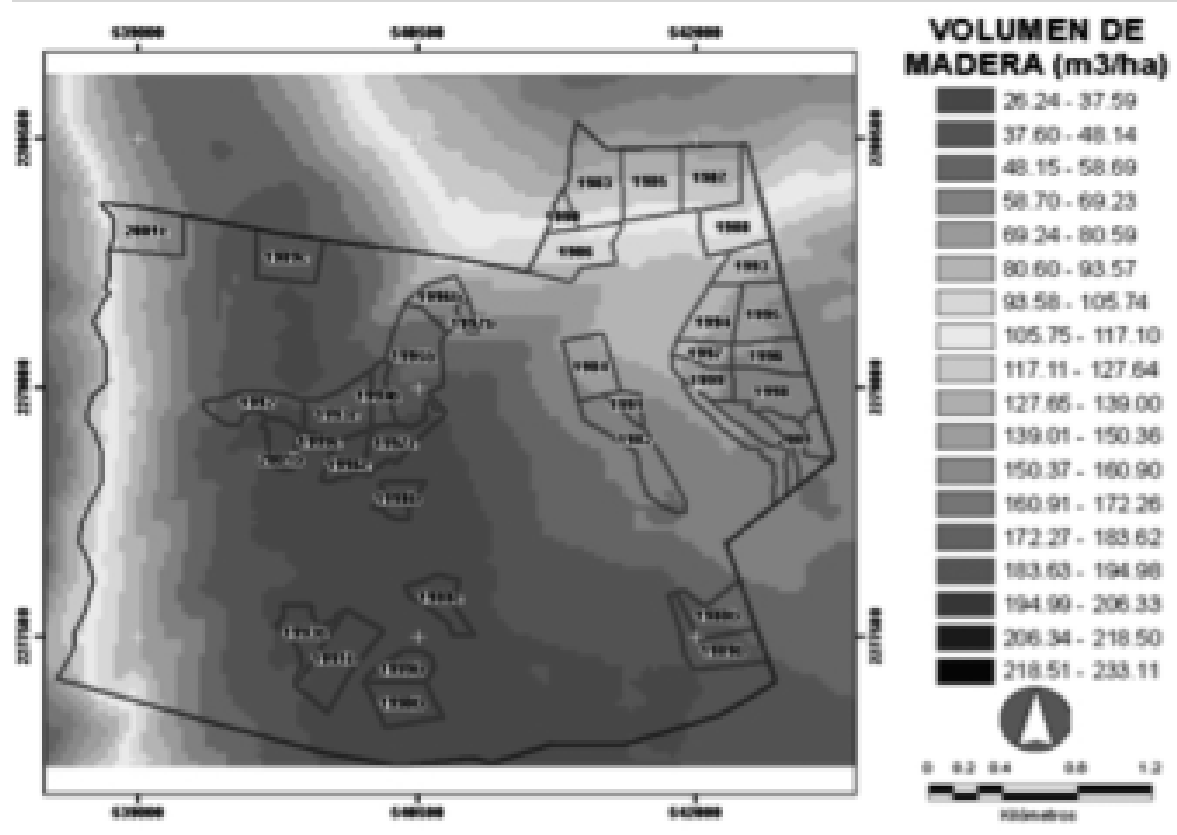

Figura 8. Distribución del volumen de madera $\left(\mathrm{m}^{3} / \mathrm{ha}\right)$ en el ejido Atopixco, Hidalgo con base en el Modelo 7 (Tabla 6). 
VOL incrementa con la ALTITUD y con el aumento en precipitación (PMA, PMAMO). Por el contrario, la cantidad de VOL disminuye al haber un aumento en la temperatura (TMAX), lo que concuerda con lo observado en los modelos 5, 6 y 7 .

Finalmente, el mayor reto al modelar el $V O L$ fue la escala de las variables cartográficas $(<1: 250000)$ de la zona de estudio. Esto produjo extensas áreas donde las variables (ALTITUD, PMA, PMAMO, PENDIENTE, TMA y TMAX) no mostraban variabilidad espacial. Como describen Fazakas et al. (1999), Franco-López et al. (2001) y Mäkelä y Pekkarinen (2001), la estimación del volumen de madera (con sensores remotos) puede aplicarse a zonas pequeñas, siempre y cuando se tenga presente que esa estimación depende del cuadrado medio del error (RMSE) de lo estimado y lo medido en campo. La pregunta relevante es ¿cómo reducir el error medio a la vez que se aumenta la varianza explicada? Al parecer las variables cartográficas son un tercio del camino, los otros dos tercios son representados por las variables biofísicas (COB, LAI5, LAIAL). Al respecto Lee et al. (1995) mostraron que es posible obtener coeficientes de correlación múltiple elevados $\left(R^{2}>0,90\right)$ si se posee información geográfica de escala adecuada. En este caso, se tenían variables categóricas de especies, edades y cobertura para toda la zona de estudio. El secreto radicó en la calidad de los mapas.

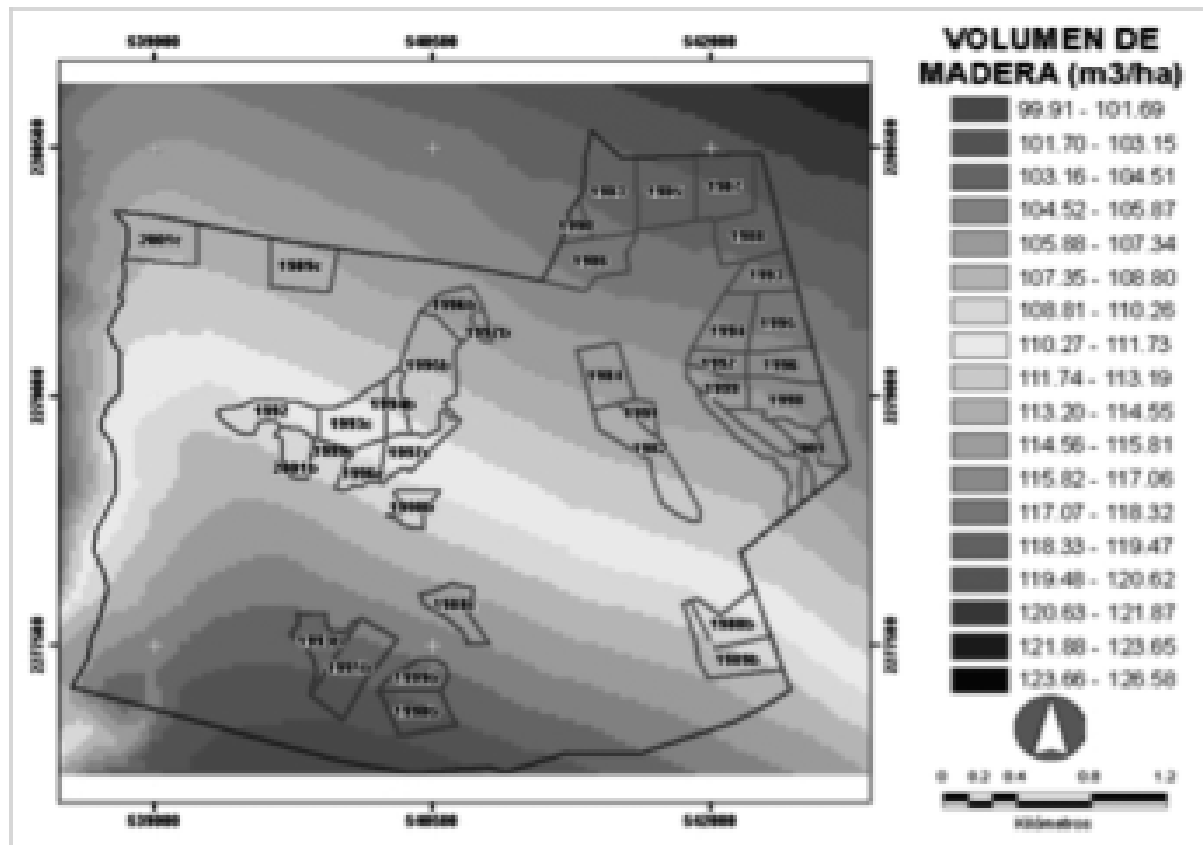

Figura 9. Distribución del volumen de madera $\left(\mathrm{m}^{3} / \mathrm{ha}\right)$ en el ejido Atopixco, Hidalgo con base en el Modelo 8 (Tabla 6). 


\section{Comparación de las estimaciones de volumen de madera: inventario vs modelación}

Todos los modelos propuestos para predecir y describir la distribución espacial del volumen de madera (VOL), presentaron sobreestimaciones con relación al inventario obtenido mediante MRR (Tabla 7). Sin desatender este punto, fue el Modelo 8 (que considera variables cartográficas) el que mejor se ajustó a la estimación de inventario total. Si se toma como referencia el inventario obtenido por MRR $\left(41453,16 \mathrm{~m}^{3}\right)$, se observa que tal estimación es similar al obtenido por el Modelo 8 (37804,99 m³). La diferencia entre ambas estimaciones es de $3648,16 \mathrm{~m}^{3}$. Por el contrario, si se toma como referencia al inventario obtenido mediante MEA, la mayoría de los modelos (5 al 7) muestran sobreestimaciones de volumen de madera, en cambio el Modelo 8 está por debajo de ese inven- tario. En este caso la diferencia entre el inventario por MEA $\left(42019,11 \mathrm{~m}^{3}\right)$ y el obtenido a través del modelo 8 (37804,99 $m^{3}$ ) es de $4214 \mathrm{~m}^{3}$.

\section{CONCLUSIONES}

La variable dasométrica área basal $(A B)$ tuvo una correlación positiva con las variables cartográficas temperatura media anual (TMA), altitud sobre el nivel medio del mar (ALTITUD) y precipitación media anual en lluvias (PMAMO). Las variables biofísicas de $C O B, L A I 5$ y $L A I A L$ también mostraron alta correlación con $A B$. Destaca la correlación de $A B$ con $\angle A I A L$, esto se explica por la relación de $A B$ con la biomasa seca del follaje, misma que fue parte importante para la estimación de LAIAL. La correlación de $A B$ con la banda 4 (B4) siempre fue negativa. Las estructuras foliares de los árboles influyeron en la relación de $A B$ con $B 4$.

Tabla 7. Comparación de las estimaciones de volumen de madera $\left(\mathrm{m}^{3}\right)$ obtenidas mediante un inventario tradicional y con inventario por SIG. Intervalos de confianza (IC) a 95\% de significancia estadística.

\begin{tabular}{|c|c|c|c|c|c|c|}
\hline \multirow[b]{2}{*}{$\begin{array}{l}\text { Superficie } \\
\text { (ha) }\end{array}$} & \multirow[b]{2}{*}{298,66} & \multicolumn{5}{|c|}{ Volumen de Madera Total } \\
\hline & & $\mathrm{m}^{3} / \mathrm{ha}$ & TOTAL & $\begin{array}{c}\text { Límite } \\
\text { inferior } \\
\text { del IC }\end{array}$ & $\begin{array}{l}\text { Límite } \\
\text { superior } \\
\text { del IC }\end{array}$ & $\begin{array}{c}\text { Precisión } \\
\%(\mathrm{~B} / \mathrm{y})\end{array}$ \\
\hline Inventario & Inventario MEA & 140,69 & 42019,11 & 39800,52 & 44237,7 & $5,28 \%$ \\
\hline Tradicional & Inventario MRR & 118,89 & 35507,37 & 33933,19 & 37081,55 & $4,43 \%$ \\
\hline Inventario & Modelo 5 (COB) & 216,54 & 64672,88 & & & \\
\hline SIG & Modelo 6 (LAI5) & 212,9 & 63585,74 & & & \\
\hline & Modelo 7 (LAIAL) & 233,11 & 69621,76 & & & \\
\hline & $\begin{array}{l}\text { Modelo } 8 \\
\text { (Cartográficas) }\end{array}$ & 126,58 & 37804,99 & & & \\
\hline \multicolumn{2}{|c|}{$\begin{array}{l}\text { COB = Cobertura arbórea } \\
\text { LAI5 = Índice de área foliar en sitio } \\
\text { LAIAL = Índice de área foliar } \\
\text { alométrico }\end{array}$} & \multicolumn{5}{|c|}{$\begin{array}{l}\text { MEA = Muestreo } \\
\text { estratificadoaleatorio } \\
\text { MRR = Muestreo de razón y } \\
\text { regresión }\end{array}$} \\
\hline
\end{tabular}


De los cuatro modelos propuestos para $A B$, el Modelo 2 ( $L A / 5)$ es el que mejor se aproximó a la estimación de inventario total. El inventario obtenido por MEA fue de $22,54 \mathrm{~m}^{2} /$ ha mientras que por Modelo 2 fue de $20,47 \mathrm{~m}^{2} / \mathrm{ha}$, la diferencia en área basal fue de $2 \mathrm{~m}^{2} / \mathrm{ha}$. Destaca el resultado obtenido con el Modelo 4 , el cual está integrado únicamente por variables cartográficas. A pesar de tener la menor $R^{2}$ ajustada $(0,7117)$ fue el segundo modelo que más se aproximó al inventario final $\left(16,09 \mathrm{~m}^{2} / \mathrm{ha}\right)$.

Para la variable dasométrica volumen de madera ( $V O L$ ) también se observó una correlación positiva con las variables TMA, ALTITUD y PMAMO. Las variables biofísicas de COB, LAI5 y LAIAL también mostraron alta correlación con VOL. Destaca la relación de VOL con SAVI.

De los cuatro modelos propuestos para VOL, el Modelo 8 (que contiene variables cartográficas) fue el que mejor se aproximó a la estimación obtenida mediante el inventario tradicional. Al tomar como referencia el inventario del predio obtenido por MRR (118,89 m3/ha) se observa que tal estimación es la más cercana al inventario obtenido por el Modelo $8\left(126,5 \mathrm{~m}^{3} / \mathrm{ha}\right)$ y la única que no sobrestima volumen total.

El mayor problema enfrentado en la modelación espacial del $A B$ y el $V O L$ fue el empleo de información de escala muy pequeña (1: 250 000). Bajo estas circunstancias, por ejemplo, la interpolación de las isotermas e isoyetas fue difícil, aparecieron amplias zonas con poca variación, razón que afectó a los modelos. En este sentido, puede afirmarse que de contar con información fisiográfica y climática de escala grande (1:25000) se habrían obtenido resultados altamente significativos (Silbernagel y Moeur, 2001). De hecho, aun trabajando con información geográfica de poca calidad, se obtuvieron coefi- cientes de determinación múltiple moderadamente altos. Razón que justifica el empleo de variables cartográficas en el inventariado de recursos forestales.

\section{REFERENCIAS}

Aguirre, C.A. 2007. Almacenamiento de carbono en bosques manejados de Pinus patula: Estimación mediante percepción remota. Tesis de Maestría en Ciencias. Colegio de Postgraduados. Postgrado Forestal. Montecillo, Texcoco, México. 98 pp.

Ángeles-Pérez, G. 1995. Efecto de la vegetación competidora en el desarrollo inicial de Pinus patula. Tesis de Maestría en Ciencias. Colegio de Postgraduados. Programa Forestal. México. 114 pp.

Ante, M.E., J.A. Peri y M.C. Serafíni. 2005. Detección de cambios a través del análisis multitemporal de imágenes satelitales en un sector de la provincia de San Luis. Publicación Monográfica INTA, EEA San Luis. 20 pp.

Barrantes, O. y C.A. Gracia. 1989. Estimación del área foliar a partir de la superficie de albura en Pinus sylvestris. Options Méditerranéennes-Série Séminaries 3:53-56.

Belsley, D.A., E. Kuh y R.E. Welsch. 2005. Regression Diagnostics: Identifying Influential Data and Sources of Collinearity. Wiley-IEEE. EUA. 201 pp.

Black, B.A. y M.D. Abrams. 2005. Disturbance history and climate response in an old-growth hemlock-white pine forest, central Pennsylvania. Journal of the Torrey Botanical Society 132 (1):103-14.

Camarero, J.J. y E. Gutiérrez. 1999. Patrón espacial de un ecotono bosque subal- 
pino-pastos alpinos (Las Cutas, Pirineos Centrales). Invest. Agr.: Sist. Recur. For. 8 (1):172-215.

Cano-Morales, E., A. Velásquez-Martínez, J.J. Vargas-Hernández, C. RodríguezFranco y A.M. Fierros-González. 1996. Área foliar específica en Pinus patula, efecto del tamaño del árbol, edad del follaje y posición en la copa. Agrociencia 30:117-122.

Carrillo-Anzurez, F., M. Acosta-Mireles y G. Tenorio-Galindo. 2004. Tabla de volumen para Pinus patula Schl. et Cham. en el Estado de Hidalgo. Folleto Técnico Núm. 2. INIFAP-SAGARPA. 16 pp.

Eastman, J.R. 2001. IDRISI for windows. User's Guide, version 3.2. Clark Labs. 144 pp.

Enciclopedia Municipios de México. 2000. Estado de Hidalgo: Zacualtipán de Ángeles.

FAO Inventario Forestal Nacional. 2004. Manual de campo modelo. Departamento de Montes, FAO (Organización de las Naciones Unidas para la Agricultura y la Alimentación). Guatemala. 89 pp.

Fazakas, Z., M. Nilsson y H. Olsson. 1999. Regional forest biomass and wood volume estimation using satellite data ancillary data. Agricultural and Forest Meteorology 98-99:417-425.

Figueroa-Navarro, C.M. 2010. Almacenamiento de carbono en bosques manejados de Pinus patula en el ejido La Mojonera, Zacualtipán, Hidalgo. Tesis de Maestría en Ciencias. Postgrado Forestal. Colegio de Postgraduados. Datos no publicados.

Franco-López, H., A.R. Ek y M.E. Bauer. 2001. Estimation and mapping of forest stand density, volume, and cover type using $k$-nearest neighbors method. Remote Sensing of Environment 77:251-274.

Frazer, G.W., C.D. Canham y K.P. Lertzman. 1999. Gap Light Analyzer (GLA), Version 2.0; Imaging software to extract canopy structure and gap light transmission indices from true-color fisheye photographs. User's manual and program documentation. Simon Fraser University, Burnaby, British Colombia, and the Institute of Ecosystem Studies, Millbrook, Nueva York.

García-García, A., F. Pérez-Cabello y J. Riva-Fernández. 2006. Evaluación de los recursos de biomasa residual forestal mediante imágenes del satélite Landsat y SIG. GeoFocus 6:205-230.

Gilabert, M.A., J. González, F.J. García y J. Meliá. 2002. A generalized soil adjusted vegetation index .Remote Sensing of Environment 82:303-310.

González-Murguía, R., E.J. Treviño, O.A. Aguirre-Calderón, J. Jiménez-Pérez, I. Cantú y R. Foroughbakhch. 2004. Rodalización mediante sistemas de información geográfica y sensores remotos. Investigaciones Geográficas UNAM 53:39-57.

Jonckheere, I., S. Fleck, K. Nackaerts, B. Muysa, P. Coppin, M. Weiss y F. Baret. 2004. Review of methods for in situ leaf area index determination Part I. Theories, sensors and hemispherical photography. Agricultural and Forest Meteorology 121:19-35.

Lee, D.C., Y.E. Shimabukuro, B.F.T. Rudorff y T. Krug. 1995. Timber volume estimation using qualitative variables. National Institute for Space Research INPE, Memorias del Congreso SELPER, México, 613-621. 
Lencinasa, J.D. y D. Mohr-Bell. 2007. Estimación de clases de edad de las plantaciones de la provincia de Corrientes Argentina con base en datos satelitales Landsat. Bosque 28(2):106-118.

Liétor-Gallego, J. 2002. Patrones de disponibilidad y limitación por nutrientes como indicadores de estado en masas de pinsapar (Abies pinsapo Boiss.). Tesis de Doctorado. Ecosistemas. España Año XI 3:1-5.

Lillesand, T.M. y R.W. Kiefer. 2001. Remote sensing and image interpretation. 4a. Ed. John Wiley and Sons, EUA. 720 pp.

Mäkelä, H. y A. Pekkarinen. 2001. Estimation of timber volume at the sample plot level by means of image segmentation and Landsat TM imagery. Remote Sensing of Environment 77:66-75.

Márquez-Linares, M. y R. Álvarez-Zagoya. 1995. Construcción de una guía de densidad para Pinus cooperi var. ornelasi con base en la clase de copa en Durango, México. Madera y Bosques 1(2):23-36.

McRoberts, R.E., M.D. Nelson y D.G. Wendt. 2002. Stratified estimation of forest area using satellite imagery, inventory data and the k-Nearest Neighbors technique. Remote Sensing Environment 82:457-468.

Miranda-Salas, M. y A.R. Condal. 2003. Importancia del análisis estadístico exploratorio en el proceso de interpolación espacial: caso de estudio Reserva Forestal Valdivia. Bosque 24(2):29-42.

Pindyck, R.S. y D.L. Rubinfeld. 1981. Econometric Models and Econometric Forecasts, $2^{a}$ ed. McGraw-Hill. EUA. 654 pp.

SAG. 1976. Inventario Forestal del Estado de Chiapas. Secretaría de Agricultura y
Ganadería. Subsecretaría Forestal y Fauna. Dirección General de Inventario Nacional Forestal. 85 pp.

Sánchez, G.A. y L. López-Mata. 2003. Clasificación y ordenación de la vegetación del norte de la Sierra Nevada, a lo largo de un gradiente altitudinal. Anales del Instituto de Biología, UNAM, Serie Botánica 74(1):47-71.

Schreuder, H.T., R. Ernst y H. RamírezMaldonado. 2005. Statistical techniques for sampling and monitoring natural resources. U.S. Department of Agriculture, Forest Service, Rocky Mountain Research Station. 154 pp.

Silbernagel, J. y M. Moeur. 2001. Modeling canopy openness and under-story gap patterns based on image analysis and mapped tree data. Forest Ecology and Management 149:217-233.

Sivanpillai, R.C., T. Smith, R. Srinivasan, M.G. Messina y X.B. Wu. 2006. Estimation of managed loblolly pine stand age and density with Landsat ETM+ data. Forest Ecology and Management 223:247-254.

Sorani V. y R. Álvarez. 1996. Hybrid maps: updating Mexico its forest cartography using Landsat TM imagery and Land use information. Geocarto International 11(4):17-23.

Trotter, C. y J. Dymond. 1997. Estimation of timber volume in a coniferus plantation forest using Landsat TM. International Journal of Remote Sensing 18(10): 2209-2223.

Valdez-Lazalde, J.R., M.J. González-Guillén y H.M. De los Santos-Posadas. 2006. Estimación de cobertura arbórea mediante imágenes satelitales multiespectrales de alta resolución. Agrociencia 40:383-394. 
Velázquez, A., J.F. Mas, G.J. Díaz, S.R. Mayorga, P.C. Alcántara, R. Castro, T. Fernández, G. Bocco, E. Ezcurra y J.L. Palacio. 2002. Patrones y tasas de cambio de uso del suelo en México. Gaceta Ecológica, INE, México, 62:21-37.

Wallerman, J. 2003. Remote sensing aided spatial prediction of forest stem volume. Doctoral thesis. Swedish University of Agricultural Sciences. Sweden. 42 pp.
Zurita-Milla, R. 2000. Determinación de variables biofísicas mediante teledetección en el marco de la agricultura de precisión. Escuela Técnica Superior de Ingenieros Agrónomos y de Montes, Universidad de Córdoba. España. $21 \mathrm{pp}$. cial de área basal y volumen de madera en bosques manejados de Pinus patula y $P$. teocote en el ejido Atopixco, Hidalgo. Madera y Bosques 16(3):75-97. 